\title{
Programas de reducción de daños en uso/abuso de drogas recreativas
}

\author{
Clara Valverde Gefaell*, Joan Pi González*y Joan Colom i Ferran** \\ *Técnico colaborador, Dirección General de Drogodependencias y Sida, Generalitat de Catalunya \\ **Director General, Drogodependencias y Sida, Generalitat de Catalunya
}

Enviar correspondencia: Joan Colom i Ferran. Departamento de Sanidad y Seguridad Social de la Generalidad de Cataluña. Travesera de les Corts 131-159. Pavelló Ave María. Barcelona. E-mail: jcolom@dsss.scs.es

\begin{abstract}
Resumen
El objetivo del presente trabajo es proponer el uso y describir los programas de reducción de daños en el uso/abuso de drogas recreativas que se están llevando a cabo desde la Dirección General de Drogodependencias y SIDA de la Generalitat de Catalunya. Las intervenciones priorizadas son las directas en los espacios de ocio para la reducción de daños con jóvenes ya consumidores. La metodología utilizada es la de iguales, en la cual jóvenes educadores dan información y apoyo a los que lo deseen. Las intervenciones se diseñan basándose en la información que facilita el Observatori de Nous Consums de Drogues en I'Ambit Juvenil en Catalunya. El objetivo principal de este tipo de intervenciones en los espacios de ocio frecuentados por jóvenes es crear diálogo y consenso entre todos los implicados (administraciones, propietarios, jóvenes, etc.) y fomentar, entre los jóvenes, una cultura del ocio inteligente y sin problemas para la salud, reduciendo los riesgos directos e indirectos del consumo en los espacios de ocio y se diseñan. En conclusión, en este artículo se propone que el trabajo en drogodependencias con jóvenes y los nuevos patrones de consumo se haga desde un óptica de Reducción de Daños, respetando y entendiendo que las sustancias son parte del ocio en nuestra sociedad y que el tiempo de ocio es un elemento clave en la construcción de la identidad de los jóvenes.
\end{abstract}

Palabras claves: jóvenes, nuevos patrones de consumo, reducción de daños, prevención.

\section{Summary}

The objective of this paper is to propose and describe the harm reduction programs employed in the use/abuse of club drugs following the recommendations of the General Direction on Drug Dependence and AIDS of the Generalitat of Catalunya. The most employed interventions were carried out in party venues in Catalonia, and made through peer-to-peer methodology in which young educators provide information and support to those desiring it. All these interventions are based on the needs-assessment information provided to us by the Observatory of New Substance Use Amongst Youth in Catalonia. Their main objective is to create dialogue and consensus amongst all concerned with "Safe Club Going" (administrations, club owners, youth, etc.). In this way we want to promote amongst youth a culture of responsible club going and to reduce the direct and indirect risks of substance use in party spaces. In conclusion, in this article we propose that interventions in the new drug use pattern among young people should be undertaken from a Harm-Reduction Approach. This means respecting and understanding that substance use is a part of leisure time activities in our society; and that leisure time is a key element in the building of one's identity/ for young people.

Key Words: youth, new patterns of substance use, Harm-Reduction. 


\section{INTRODUCCIÓN}

Hay un aumento en la preocupación social y sanitaria sobre el consumo de sustancias entre jóvenes en los espacios nocturnos, los "nuevos patrones de consumo", ya que ha habido cambios en la forma de divertirse de los jóvenes ("ir a tope" y que los fines de semana que son más largos), en las sustancias (la aparición del éxtasis y otras drogas de síntesis) y en la manera que se consumen (mezclas para potenciar y aguantar el ritmo de la noche).

Entendemos que los patrones de ocio de los jóvenes son el producto de numerosos factores sociales complejos: el consumismo, la masificación, el aumento del paro, la prolongación de la adolescencia y la nueva cultura del ocio.

Los nuevos patrones de consumo de sustancias recreativas son una actividad más, entre otras (y no necesariamente la más importante), en los estilos de vida y en las diferentes formas de vivir e interpretar el tiempo libre, que son parte del proceso de socialización y diferenciación de los jóvenes.

Los espacios y el tiempo de ocio son el marco principal en el cual los jóvenes construyen y reelaboran su identidad, ya que otros ámbitos, como el laboral, han perdido la importancia que tenían antes en el proceso de incorporación al mundo adulto. Los espacios de ocio se convierten en ámbitos de identificación de los diferentes grupos, configurando espacios simbólicos donde las normas y comportamientos se consideran propios a su estilo generacional, fuera del control de los adultos.

Los factores comunes entre los diferentes grupos de jóvenes son:

- la importancia de compartir características y símbolos comunes como señal de identificación

- la importancia de la noche como tiempo de diversión, de ruptura con el tiempo cotidiano, de nuevas experiencias y de asumir ciertos riesgos
- la importancia de la música, el baile y el consumo de ciertas drogas que faciliten la diversión, la desinhibición, la comunicación (en algunos espacios es más importante el lenguaje del cuerpo que la comunicación verbal) y la prolongación de la fiesta

\section{ENFOQUE}

Aún con la complejidad sociológica de este fenómeno, creemos que es posible y muy necesario el intervenir para asegurarnos la salud y la seguridad de los jóvenes con un enfoque de reducción de daños.

Trabajamos con la realidad: el consumo de sustancias en los espacios de ocio no es un fenómeno pasajero, nos guste o no.

Para que nuestras intervenciones sean posibles, es necesario encontrar el equilibrio entre dar información útil sobre los riesgos y el respetar la cultura de los jóvenes. Cualquier mensaje o material que frivoliza o ridiculiza la cultura de este sector de la sociedad, será ineficaz. Igualmente, si en los mensajes o tipos de intervención, los jóvenes se sienten juzgados, los esfuerzos de los programas serán en balde.

En estos programas que llevamos a cabo desde la Dirección General de Drogodependencias y Sida de la Generalitat de Catalunya, nos esforzamos por respetar y potenciar la inteligencia y las habilidades de los jóvenes. De esta manera, nuestro objetivo principal, el de fomentar cambios de actitudes y conductas, es posible.

\section{OBJETIVOS}

\subsection{Con los jóvenes}

a. fomentar una cultura del ocio inteligente y sin problemas para la salud

b. reducir los riesgos directos (sobredosis y desarrollo de adicciones) e indirectos (acci- 
dentes viales y violencia) relacionados con el consumo en los espacios de ocio

c. aumentar, entre los jóvenes, la toma de decisión responsable para el auto-protección ante el consumo y el ocio

d. aumentar la información disponible sobre las sustancias utilizadas en el ámbito del ocio.

\subsection{Con los padres}

a. fomentar las habilidades de comunicación y negociación con sus hijos

b. aumentar los conocimientos sobre el enfoque de reducción de daños en el tema del consumo de sustancias.

\subsection{Con los propietarios de los estableci- mientos de ocio}

a. consensuar el "Safe Clubbing" (el aumentar las medidas de salud y seguridad en los establecimientos de ocio)

b. continuar la colaboración en intervenciones de información y reducción de daños con los que frecuentan estos establecimientos

c. aumentar la formación con los responsables de los establecimientos sobre temas de salud y seguridad

\subsection{Con las unidades de urgencias hospi- talarias}

a. continuar el sistema de notificación rápida de intoxicaciones por el uso de sustancias de ocio

b. aumentar las habilidades de los profesionales de estas unidades para llevar a cabo el "Counselling" con los jóvenes ingresados por intoxicación por sustancias de ocio

c. facilitar la distribución de material de reflexión y prevención específico a jóvenes (y su entorno) que han sufrido una intoxicación por sustancias de ocio.

\section{INTERVENCIONES}

\section{1 Entre iguales}

Las intervenciones que priorizamos son las directas en los espacios de ocio para la reducción de daños con jóvenes que ya consumen sustancias. Ellos son nuestra prioridad para intentar dotarlos de las habilidades e información necesarias en este ambiente. La metodología ideal es la de entre iguales, en la cual jóvenes que conocen el ambiente de intervención son formados como agentes de salud. Su mejor herramienta es el diálogo que complementan con información y materiales relevantes. En ningún momento estos agentes de salud interfieren con el ambiente de fiesta ni intervienen con personas que no lo han solicitado. Con el permiso de los encargados del espacio de fiesta, están disponibles para los jóvenes que quieran dialogar, tengan preguntas o necesiten ayuda.

Con esta metodología, se llevan a cabo varias intervenciones:

\section{1.a. Primera intervención éxtasis}

En 1996 y 1997 se llevó a cabo importantes intervenciones de información en los espacios de ocio en Catalunya sobre los riesgos que conlleva el consumo de éxtasis. En total se distribuyeron 300.000 tarjetas informativas, 500 camisetas y se hicieron 3022 cuestionarios para evaluar la eficacia de los mensajes de las tarjetas. Esto se hizo en setenta locales musicales en treinta y nueve municipios. La evaluación de esta intervención y la valoración positiva de esta, nos reafirmó la necesidad de seguir trabajando en esta línea.

Para complementar esta intervención, se elaboró un documento para médicos de atención primaria para darles información y herramientas para poder intervenir ante consultas sobre el tema del éxtasis. 


\section{1.b. Som.Nit}

El proyecto Som. Nit ("somos noche" en castellano) se comenzó en el 2000, conjuntamente con la Secretaría General de Juventud y Creu Roja y es el ejemplo del tipo de intervención que creemos más acertada en el ámbito del consumo en los espacios de ocio. Este proyecto interviene directamente, entre iguales, con información, apoyo y análisis de pastillas de éxtasis con el fin de educar a los que frecuentan estos espacios sobre las sustancias, sus riesgos y sobre las decisiones necesarias para reducir los peligros y daños del consumo.

En la primera fase, se hizo un análisis de la realidad en el ámbito de las discotecas de Catalunya con la cual se pudo enfocar la intervención.

La intervención consiste en que educadores forman equipos de agentes de salud voluntarios que intervienen en discotecas, fiestas y raves. Se han formado, en estos tres años, 477 voluntarios por toda Catalunya que han intervenido haciendo 244 salidas.

Estos equipos de agentes apoyan sus intervenciones con un material elaborado especialmente para el proyecto Som.nit. Se hicieron tarjetas pequeñas con información sobre una sustancia en cada una: cocaína, éxtasis, cannabis, ketamina, LSD, speed, alcohol y GHB. Otra serie de tarjetas tienen como objetivo la reflexión en el proceso de toma de decisiones ante el consumo. Cada una de estas tarjetas tiene una pregunta o consejo después del cual se lame un círculo que deja ver el mensaje educativo. La tercera serie de tarjetas, son unas "postal-free" con monólogos de jóvenes sobre el problema de consumir cuando se está en un estado emocional bajo y dos sobre las sensaciones y malestares después de un consumo excesivo. Se han distribuido 158.858 tarjetas.

\section{1.c. Grupo 'Energy Control'}

Este grupo son los pioneros de las intervenciones de reducción de daños en los espacios de ocio. Es un programa de carácter comunitario que lleva a cabo intervenciones preventivas en espacios de ocio y depende de la Asociació Benestar i Desenvolupament (ABS). Se basan en un conocimiento directo de la realidad y elaboran, editan y reparten material preventivo en el estilo de la cultura de los jóvenes para conseguir la credibilidad y aceptación necesarias. Colaboran con diferentes "actores de la noche" (DJs, propietarios de discotecas, promotores de festivales, etc) para introducir un enfoque de reducción de daños en sus mensajes.

Aparte del trabajo que hacen en los espacios de ocio, están disponibles a los jóvenes a través de una línea telefónica y de una página web.

Energy Control también tiene un papel importante en formación de profesionales y de informar a la sociedad en general a través de los medios de comunicación.

\section{1.d. Summer Campaign}

Dado el alto número de jóvenes turistas europeos que frecuentan el litoral catalán que, en el contexto lúdico de las vacaciones pueden llevar a cabo consumos de riesgo, en el verano del 2002 se ha colaborado en una iniciativa específica a esta población. A través de la metodología entre iguales con jóvenes de varios países europeos, se ha intervenido en francés, inglés, flamenco, alemán, casteIlano y catalán en zonas turísticas para intentar prevenir el consumo de riesgo de alcohol y de otras sustancias entre jóvenes turistas de 18 a 25 años.

En la Summer Campaign han colaborado 40 jóvenes como agentes de salud procedentes de Bélgica, Alemania, Inglaterra y España que dialogaron con jóvenes turistas de su mismo país para informar, motivar y fomentar la reflexión sobre el consumo de sustancias recreativas durante las vacaciones.

\section{1.e. Europeers}

Este proyecto europeo está dirigido a padres y jóvenes. Uno de sus objetivos es el promover la reflexión sobre el papel de los 
padres y madres en la formación de sus iguales en temas de prevención y promoción de hábitos. Otro objetivo es el formar a líderes jóvenes en el tema del riesgo y la reducción de daños en drogodependencias.

\subsection{Grupo de Trabajo "Nuevos Consumos en los Espacios de Ocio"}

Para aumentar la eficacia de las intervenciones en el ámbito del consumo de sustancias recreativas, es importante la coordinación y la colaboración entre todas las instituciones y organizaciones relevantes. Con este fin, en el año 2000, desde la Dirección General de Drogodependencias y Sida de la Generalitat de Catalunya, se creó el grupo de trabajo "Nuevos Consumos en los Espacios de Ocio" compuesto por administraciones y un amplio abanico de organizaciones y profesionales relacionados al ámbito juvenil y de las drogodependencias. La finalidad del grupo es de compartir, desarrollar y coordinar ideas para intervenir en este ámbito para reducir los peligros y riesgos.

\subsection{Observatorio}

Para asegurarnos que nuestro trabajo responde a la realidad siempre cambiante, nos apoyamos en el 'Observatori de Nous Consums de Drogues' en I'Ambit Juvenil, que desde 1999 nos da una información muy valiosa sobre la situación de nuevos consumos en Catalunya. El Observatorio es un sistema de información rápida y sensible, con el objetivo de detectar los patrones de consumo de sustancias emergentes en sus fases iniciales y la estabilidad relativa del consumo de las sustancias conocidas o los cambios que se puedan producir en estas. Esta información está acompañada de un análisis socio-antropológico para mejor poder enfocar las intervenciones.

\subsection{Smart shops}

Unas nuevas sustancias recreativas son las llamadas "inteligentes" o "eco-drogas" que son parte de ciertas corrientes culturales europeas que responden a un interés por lo natural. Estas sustancias naturales se utilizan para conseguir ciertos estados o efectos deseados y están disponibles por internet y en establecimientos llamados "Smart Shops".

Estas sustancias, que no están en las listas internacionales de fiscalización de sustancias psicotrópicas, en el Estado Español presentan problemas ya que hay un vacío legal ante la falta de una normativa actualizada que rija la actividad de herboristerías y dietéticas. Al mismo tiempo algunas de estas sustancias podrían tener efectos negativos para la salud $y$, también, las etiquetas a menudo están sólo en inglés. Ante esto, la Agencia Española del Medicamento, atendiendo a la Ley del Medicamento, ha decidido ir retirando del mercado la mayoría de los productos que venden las Smart Shops.

Aún con esta situación ambigua, desde la Dirección General, se pensó que era necesario hacer una intervención de reducción de daños sobre estas sustancias. Sabíamos, por información del Observatorio, que los usuarios de estas sustancias no son por norma general personas marginales o autodestructivas, si no personas en su mayoría normativas e interesadas por su salud. Por esto pensamos que sería un público receptivo a una campaña de reducción de daños. Como estrategia decidimos elaborar un material sobre los productos "inteligentes" para estos usuarios que fuera distribuido en las Smart Shops. Para aumentar la eficacia de esta intervención, se hizo un curso de formación para los propietarios y dependientes de estos establecimientos en materia de reducción de daños, comunicación y otros temas relevantes. Participaron 25 personas y duró 16 horas.

El material distribuido titulado "Smart Drugs" se realizó con el grupo Energy Control y tras consultar con representantes de las Smart Shops y trataba los temas de las sustancias "inteligentes" y sus efectos, infor- 
mación clave para cuestionar creencias relacionadas con estos productos (que lo legal es menos perjudicial y que lo natural es saludable), fomentaba la toma de decisiones responsables (quien es inteligente es la persona no la sustancia) y recomendaciones a tener en cuenta ante el consumo. Hasta septiembre del 2002, se han repartido 5.000 ejemplares de este material.

\subsection{Formación}

Para formar a los profesionales sociosanitarios de los servicios de la red de drogodependencias, de Atención Primaria y de otros ámbitos, se ofrece una formación sobre las sustancias recreativas, los nuevos patrones de consumo y las intervenciones relevantes.

\subsection{Mesa de consenso sobre la salud y seguridad en los espacios de ocio noctur- no y festivales}

En el año 2002 se ha incrementado la preocupación social sobre los jóvenes y el consumo de ocio. En respuesta, el anterior Conseller de Sanitat, Eduard Rius, presentó al Parlament de Catalunya en junio, una serie de iniciativas que la Direcció General de Drogodependéncies i Sida está llevando a cabo para disminuir los problemas relacionados a la salud y la seguridad de los jóvenes en los espacios de ocio, sobretodo en el consumo de sustancias. De estas iniciativas, la más importante es la del "Safe Clubbing". Esto es un concepto desarrollado en el Reino Unido y es un enfoque desde todas las posibles vertientes para que los espacios de ocio sean más seguros. Para esto, la D.G. de Drogodependencias y Sida ha organizando una mesa de consenso en la cual administraciones, el sector de las discotecas y los otros agentes implicados por este tema, se reúnen para llegar a un acuerdo sobre el tipo de medidas necesarias para reducir los posibles problemas en los espacios de fiesta. Los temas sobre los cuales se están desarrollando ideas son: los aforos (control del movimiento de masas en los espacios de ocio), dispositivos técnicos (ventilación, etc), seguridad (vigilancia y resolución de conflictos), salud (prevención de urgencias, detección precoz de problemas, "chill-out" o espacio de descanso, información para la reducción de daños, etc.)

Aparte de la mesa de consenso se está haciendo un trabajo adicional de información y diálogo con las discotecas para mejor desarrollar este acuerdo.

\subsection{Iniciativa "Por un noche más segura"}

Se está elaborando una serie de iniciativas, dirigida a los jóvenes entre 16 y 30 años que acuden a los espacios de ocio nocturno con el objetivo de promover conductas responsables ante el consumo y evitar que el uso experimental o ocasional se convierta en un uso problemático.

Se están desarrollando carteles para discotecas, una guía para los usuarios de las discotecas sobre el consumo responsable, camisetas y otros materiales. También se elaborará un spot para la salas de cine sobre este tema. Esto se complementará con una página web para que los jóvenes tengan más información.

\subsection{Urgencias}

Se ha hecho un trabajo estrecho con las unidades de urgencias de los hospitales de Catalunya para la notificación de casos de intoxicación por uso de las nuevas sustancias de ocio. Dentro de esta colaboración se ha elaborado un material para que el profesional de urgencias pueda distribuir al joven ingresado por el abuso de las nuevas sustancias y el policonsumo. Este material tiene como objetivo informar y prevenir que se repitan las situaciones de riesgo. En enero del 2003 se llevará a cabo una formación de profesionales de urgencias sobre el tema de cómo comuni- 
carse y trabajar con jóvenes que ingresan por consumo excesivo de sustancias.

\section{CONCLUSIÓN}

El comportamiento de los jóvenes en los espacios de ocio y, en particular, con las sustancias que existen, es parte de factores sociales múltiples y complejos. Pero el consumo de sustancias como parte del ocio es una realidad que no parece estar disminuyendo en ningún país desarrollado. Esto requiere seguir haciendo un trabajo cuidadoso y a largo plazo de educar y ayudar a responsabilizar los jóvenes, sus padres, los propietarios de los locales de ocio y todos los otros agentes implicados en nuestra sociedad.

\section{REFERENCIAS}

Calafat A. (1997). La Representación Social de las Drogas de Diseño en Europa. En: XXIV Jornadas Nacionales Socidrogalcohol. Libro de Actas: 39-61. Valencia: Generalitat Valenciana.

Díaz A, Pallarés J, Barruti M. (2000). Primer informe. Observatori de nous consums de drogues en I'àmbit juvenil. Barcelona: Insititut Genus (Documento fotocopiado de difusión limitada).

Díaz A, Pallarés J, Barruti M. (2001). Observatori de nous consums de drogues en l'àmbit juvenil. Barcelona: Insititut Genus (Documento fotocopiado de difusión limitada).
EMCDDA.(1998). The use of amphetamines, ecstasy and LSD in the European Community: A review of data consumption patterns and current epidemiological literature. Londres: EMCDDA.

Gamella JF, Álvarez-Roldán A. (1997). Drogas de síntesis en España. Patrones y tendencias de adquisición y consumo. Madrid: Plan Nacional sobre Drogas.

Geertz C. (1987). La interpretación de las culturas. Barcelona: Gedisa.

Klee H. (1995). Amphetamine-misusing groups: A feasability study of the use of peer group leaders for drug prevention work among their associates. Home Office Drug Prevention Initiative. Paper 3. Londres: Home Office.

Lenton S, Boys A. (1997). Raves, drugs and experience: drug use by a simple or people who attend raves in Western Australia. Addiction, 2: 1327.

National Institute on Drug Abuse. (1990). The indigenous leader outreach model: Intervention manual. Washington DC: US Department of Health and Human Services.

Romaní O. (1999). Las drogas, sueños y razones. Barcelona: Ariel.

Romaní O. (1995). Intervención comunitaria en drogodependencias. Etnografía y sentido común. Toxicodependencias, 2:33-46.

Som.Nit. (2001). Projecte d'acció directa de prevenció de riscos en el consum de drogues adreçat a joves en espais d'oci nocturn. Barcelona: Creu Roja Juventud, Direcció General de Drogodependències i Sida de la Generalitat de Catalunya y Secretaría General de Juventud de la Generalitat de Catalunya.

Strunin L, Hingson R. (1992). Alcohol, drugs and adolescent sexual behaviour. Int J Addict, 27:129-146. 
